\title{
PRIME FACTORIZATION AND DOMINATION IN THE HIERARCHICAL PRODUCT OF GRAPHS
}

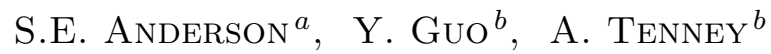 \\ AND \\ K.A. $\mathrm{WASH}^{b}$ \\ ${ }^{a}$ Department of Mathematics \\ University of St. Thomas \\ St. Paul, MN USA \\ ${ }^{b}$ Department of Mathematics \\ Trinity College, Hartford, CT USA \\ e-mail: ande1298@stthomas.edu, \\ yaoqi.guo, asa.tenney, \\ kirsti.wash@trincoll.edu
}

\begin{abstract}
In 2009, Barrière, Dalfó, Fiol, and Mitjana introduced the generalized hierarchical product of graphs. This operation is a generalization of the Cartesian product of graphs. It is known that every connected graph has a unique prime factor decomposition with respect to the Cartesian product. We generalize this result to show that connected graphs indeed have a unique prime factor decomposition with respect to the generalized hierarchical product. We also give preliminary results on the domination number of generalized hierarchical products.
\end{abstract}

Keywords: generalized hierarchical product, Cartesian product, prime factor decomposition.

2010 Mathematics Subject Classification: 05C70, 05C76.

\section{REFERENCES}

[1] M. Azari and A. Iranmanesh, Chemical graphs constructed from rooted products and their Zagreb indices, MATCH Commun. Math. Comput. Chem. 70 (2013) 901-919.

[2] L. Barrière, F. Comellas, C. Dalfó and M.A. Fiol, The hierarchical product of graphs, Discrete Appl. Math. 157 (2009) 36-48.

doi:10.1016/j.dam.2008.04.018 
[3] L. Barrière, C. Dalfó, M.A. Fiol and M. Mitjana, The generalized hierarchical product of graphs, Discrete Math. 309 (2009) 3871-3881.

doi:10.1016/j.disc.2008.10.028

[4] B. Brešar, P. Dorbec, W. Goddard, B. Hartnell, M.A. Henning, S. Klavžar and D. Rall, Vizing's conjecture: A survey and recent results, J. Graph Theory 69 (2012) $46-76$. doi:10.1002/jgt.20565

[5] J.F. Fink, M.S. Jacobson, L.F. Kinch and J. Roberts, On graphs having domination number half their order, Period. Math. Hungar. 16 (1985) 287-293. doi:10.1007/BF01848079

[6] C.D. Godsil and B.D. McKay, A new graph product and its spectrum, Bull. Aust. Math. Soc. 18 (1978) 21-28. doi:10.1017/S0004972700007760

[7] R. Hammack, W. Imrich and S. Klavžar, Handbook of Product Graphs, Second Edition (CRC Press, Boca Raton, FL, 2011).

[8] M.S. Jacobson and L.F. Kinch, On the domination of the products of graphs II: Trees, J. Graph Theory 10 (1986) 97-106. doi:10.1002/jgt.3190100112

[9] S. Jung, S. Kim and B. Kahng, Geometric fractal growth model for scale-free networks, Phys. Rev. E 65 (2002) 056101. doi:10.1103/PhysRevE.65.056101

[10] K.M. Koh, D.G. Rogers and T. Tran, Products of graceful trees, Discrete Math. 31 (1980) 279-292. doi:10.1016/0012-365X(80)90139-9

[11] D. Kuziak, I.G. Yero and J.A. Rodriguez-Velazquez, Strong metric dimension of rooted product graphs, Int. J. Comput. Math. 93 (2016) 1265-1280. doi:10.1080/00207160.2015.1061656

[12] A. Meir and J.W. Moon, Relations between packing and covering numbers of a tree, Pacific J. Math. 61 (1975) 225-233. doi:10.2140/pjm.1975.61.225

[13] M.E.J. Newman, The structure and function of complex networks, SIAM Rev. 45 (2003) $167-256$. doi:10.1137/S003614450342480

[14] G. Sabidussi, Graph multiplication, Math. Z. 72 (1960) 446-457. doi:10.1007/BF01162967

[15] V.G. Vizing, The cartesian product of graphs, Vyčisl. Sistemy 9 (1963) 30-43, in Russian. English translation in: Comp. El. Syst. 2 (1966) 352-365.

Received 24 September 2015

Revised 21 June 2016

Accepted 6 July 2016 